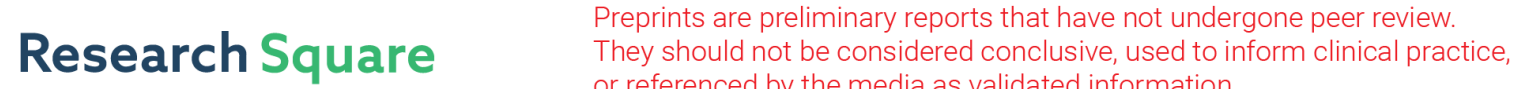 or referenced by the media as validated information. \\ Exploring the Mechanism of Laminaria for the Treatment of Alzheimer's Disease based on Network Pharmacology
}

\section{Le Yu}

Sichuan International Studies University

\section{Shuchen Pei}

Chongqing University of Science and Technology

\section{Kangyao Yuan}

Chongqing University of Science and Technology

\section{Jian Zhang}

Sichuan International Studies University

\section{Jingya Zhao}

Sichuan International Studies University

Shuiqin Chai ( $\square$ chai0104sq@126.com )

Chongqing University of Science and Technology - New Campus: Chongqing University of Science and Technology https://orcid.org/0000-0002-3072-0557

\section{Research}

Keywords: Alzheimer's disease, Laminaria, network pharmacology, mechanism

Posted Date: March 2nd, 2021

DOl: https://doi.org/10.21203/rs.3.rs-250110/v1

License: (c) (i) This work is licensed under a Creative Commons Attribution 4.0 International License. Read Full License 


\section{Abstract}

Background $\mathbb{L}$ Laminaria japonica has also been reported to have a therapeutic effect on $A D$, but the mechanism is not entirely clear. To explore the mechanism of Laminaria for the treatment of Alzheimer's disease (AD), the "active components-targets" network and the protein-protein interaction (PPI) network were constructed for analyzing targets' functions and pathways.

Methods $₫$ The main active components of Laminaria were extracted using the TCMSP database and were predicted and screened by GeneCards. Cytoscape was used to construct the "drug-components-targetsdisease" network. STRING and Cytoscape were applied to map the PPI network. The Gene Ontology (GO) terms and KEGG pathways of targets were analyzed by Metascape.

Results: Seven active components involving 23 active targets were obtained. The network analysis elucidated that Laminaria was mainly involved in cell process, metabolic process, response to stress and other biological processes. CASP3, PPARG, RELA, CCND1 and CASP9 played a key role in treating AD by regulating two small cell lung cancer and Toxoplasmosis.

Conclusion: This study demonstrated that Laminaria could prevent and treat AD with advantages of multi-components, multi-targets and multi-pathways, which explored a new way for further research on the mechanism of Laminaria in the treatment of $A D$.

\section{Background}

Alzheimer's disease (AD), characterized by impairment of memory, cognitive dysfunction and social disorders, is a chronic neurodegenerative disease[1]. According to Alzheimer's Disease International, dementia affects 50 million people worldwide, with a new case of dementia occurring somewhere in the world every 3 seconds. The symptoms of the disease is most common in those aged 60 or older and the neurologic changes caused by $A D$ are irreversible[2]. The aging of the global population is unprecedented. The number of people over 60 in the world is projected to increase by 56\% between 2015 and 2030, and by 2050 the global elderly population is projected to more than double. Thus, age remains the greatest risk factor for $A D$. The pathological hallmarks of $A D$ include amyloid $\beta$-protein deposition[3], abnormal phosphorylation of the protein tau[4], neuroinflammatory response[5], cholinergic deficit[6], oxidative stress[7], et al. At present, medicine for treatment of $A D$ can only relieve the symptoms and have relatively large side effects, which cannot repair nerve damage and prevent the deterioration of the disease[8-10]. Therefore, it is urgent to systematically elucidate the mechanism of $A D$ and find safe and effective agents against $A D$.

Laminaria japonica, the most common member of the brown algae family, is not only well-known as "longevity food", but also as traditional Chinese herbal medicine that is used to prevent and treat various diseases for over a thousand years[11]. Laminaria is rich in vitamins, minerals, dietary fiber, proteins and polysaccharides[12], which has shown to possess many biological activities such as antibacterial[13], antiviral[14], anti-inflammatory[15], anti-tumor[16], anti-diabetes[17] and antioxidant[18] and protective 
effects against liver damage, hypertension, obesity, insomnia[19]. Recently, Laminaria has also been reported to have a therapeutic effect on $A D$, but the mechanism is not entirely clear.

Network pharmacology is an emerging approach to explore the relationship between drugs and diseases that integrates system biology, network analysis, bioinformatics and multi-directional pharmacology[20]. It devotes to understand the drug's pharmacological mechanism and development in the network perspective. Network pharmacology can quickly and efficiently analyzes the mechanism of traditional Chinese medicine in a modern way[21]. In this study, the "drugs-components-targets-disease" network was constructed to analyze the relationship between components of Laminaria and AD-related target proteins to investigate the binding affinity and predict the possible binding sites of drugs. This strategy could provide a reasonable basis for further clinical and experimental research in Laminaria's action mechanism against AD.

\section{Materials And Methods}

\section{Collection and selection of main chemical components}

The chemical components of Laminaria were collected from the Traditional Chinese Medicine Systems Pharmacology (TCMSP) database and analysis platform (http://tcmspw.com/tcmsp.php)[22]. Oral bioavailability $(\mathrm{OB})$ is an important pharmacokinetic parameter in drug absorption, distribution, metabolism and excretion, indicating the rate and degree of systemic absorption of active ingredients in oral drugs[23]. The parameters of $O B \geq 30 \%$ are considered to be absorbed and utilized by the body. Drug-likeness $(\mathrm{DL})$ is a necessary condition for preparing compound medicine, the value of which indicates the similarity between the ingredients and known chemical drugs. $D L \geq 0.18$ is generally considered as an important reference for the activity[24]. According to the recommended criterion in TCMSP database, $\mathrm{OB} \geq 30 \%$ and $\mathrm{DL} \geq 0.18$ were used to select the active components.

\section{Collection of potential targets for Alzheimer's disease}

The targets of potentially active components were collected in the TCMSP database. The collected targets were imported into the Uniprot database (http://www.uniprot.org/) by name with the "Homo sapiens" setting to obtain human-related protein targets. Different ID types of the proteins were converted to UniProt IDs. The GeneCards (https://www.genecards.org/) database was used to obtain the gene name of each target by searching the keyword "Alzheimer's Disease"[25].

\section{Construction of the "drug-targets-disease" interaction network}

The targets of active components of Laminaria and the targets of AD were imported into a website called Bioinformatics \& Evolutionary Genomics (http://bioinformatics.psb.ugent.be/webtools/Venn/), and the 
overlapping targets were collected. The target-disease and component-target network was created and merged to construct the component-target-disease network model using Cytoscape 3.7.0 software[26].

\section{Construction of PPI network}

STRING 10.5 (https://string-db.org/) is a database of known and predicted protein-protein interactions including direct (physical) and indirect (functional) associations[27]. The AD-related common targets and the potential targets of Laminaria were applied to construct the PPI network using STRING 10.5 database with the "Homo sapiens" setting to achieve a comprehensive understanding of the relationships among compounds, targets, and AD. The STRING automatically scores each PPI, and the higher the score, the higher the confidence. In this study, a PPI network consisting of the products of gene expression was constructed based on the 30 data with top scores.

\section{Analysis of targets' pathways}

To further understand the function of targets' application in the signal pathway, the Laminarin-AD overlapping targets were introduced into the Metascape database (https://metascape.org/), in which these targets were standardized under the "Homo sapiens" setting and the threshold was set as $P \leq 0.05$. Gene Ontology (GO) terms[28] and Kyoto Encyclopedia of Genes and Genomes (KEGG)[29] pathways were analyzed. The pathway map of the effect of Laminaria on AD was constructed by KEGG Mapper (https://www.kegg.jp/). GraphPad Prism 5.0 was used for mapping.

\section{Results}

\section{Screening of active compounds}

A total of 48 compounds in Laminaria were obtained from TCMSP. Based on the absorption, distribution, metabolism, excretion calculation, 7 active compounds with $\mathrm{OB} \geq 30 \%$ and $\mathrm{DL} \geq 0.18$ were screened. The information of the 7 active compounds in Laminaria was shown in Table 1. 
Table 1

Main active compounds in Laminaria

\begin{tabular}{|lllcl|}
\hline NO. & MOL ID & Name & OB \% & DL \\
\hline 1 & MOL010615 & Saringosterol & 43.48 & 0.62 \\
\hline 2 & MOL010616 & Eckol & 87.06 & 0.63 \\
\hline 3 & MOL010617 & Eicosapnte Macnioc Acid (1553-41-9) & 45.66 & 0.21 \\
\hline 4 & MOL010625 & 24-Methylenecholesterol & 43.54 & 0.76 \\
\hline 5 & MOL001439 & Arachidonic acid & 45.57 & 0.2 \\
\hline 6 & MOL000953 & CLR & 37.87 & 0.68 \\
\hline 7 & MOL009622 & Fucosterol & 43.78 & 0.76 \\
\hline
\end{tabular}

\section{Identification of the targets of Laminaria on AD}

A total of 72 protein targets were collected based on the above 7 active compounds. 34 AD-related targets were obtained when the 72 targets were mapped to the UniProt database for normalizing and standardizing naming. There are 8,718 AD-related targets in the GeneCards database. The targets of drug components were compared with that of $A D$ and 23 potential targets related to the treatment of $A D$ with Laminaria were selected (Table 2). 
Table 2

Information on potential anti-AD drug targets from Laminaria

\begin{tabular}{|c|c|c|}
\hline No. & Target & Symbol \\
\hline 1 & Trypsin-1 & PRSS1 \\
\hline 2 & Coagulation factor VII & F7 \\
\hline 3 & Estrogen receptor beta & ESR2 \\
\hline 4 & Apoptosis regulator $\mathrm{Bcl}-2$ & BCL2 \\
\hline 5 & Caspase-9 & CASP9 \\
\hline 6 & Caspase-3 & CASP3 \\
\hline 7 & Prostaglandin G/H synthase 1 & PTGS1 \\
\hline 8 & Progesterone receptor & PGR \\
\hline 9 & Mineralocorticoid receptor & NR3C2 \\
\hline 10 & Retinoic acid receptor RXR-gamma & RXRG \\
\hline 11 & Transcription factor p65 & RELA \\
\hline 12 & G1/S-specific cyclin-D1 & CCND1 \\
\hline 13 & Peroxisome proliferator-activated & PPARG \\
\hline 14 & Tumor necrosis factor receptor superfamily member $1 \mathrm{~A}$ & TNFRSF1A \\
\hline 15 & Arachidonate 5-lipoxygenase & ALOX5 \\
\hline 16 & P-selectin & SELP \\
\hline 17 & Beta-galactosidase & GLB1 \\
\hline 18 & ATP-binding cassette sub-family A member 1 & ABCA1 \\
\hline 19 & Mitochondrial uncoupling protein 2 & UCP2 \\
\hline 20 & Complement $\mathrm{C} 1 \mathrm{r}$ subcomponent & $\mathrm{C} 1 \mathrm{R}$ \\
\hline 21 & Cholesteryl ester transfer protein & CETP \\
\hline 22 & ATP-binding cassette sub-family G member 1 & ABCG1 \\
\hline 23 & Multidrug resistance-associated protein 4 & ABCC4 \\
\hline
\end{tabular}

\section{Construction and analysis of the "drug-components-targets- disease" network}


Information about Laminaria's active components and overlapping targets was imported into Cytoscape 3.7.0 to establish a "drug-components-targets-disease" visualization network. As shown in Figure 1, the purple node represented the drug thallus Laminariae, the yellow nodes represented the active components, the green nodes represented overlapping genes between Laminaria and AD, and the pink node represented the disease AD. The same target corresponded to different active components, and vice versa, which sufficiently suggested Laminaria's characteristics, multi-components and multi-targets.

\section{Construction and analysis of the PPI network}

The PPI network was constructed when the target proteins were introduced into the STRING database and their names were standardized under the "Homo sapiens" setting. As shown in Table 2, one node represented one protein, and the edge between the two nodes indicated the interaction between proteins. It was speculated that CASP3, PPARG, RELA, CCND1, CASP9 were the key targets for the treatment of AD with Laminarin.

\section{Analysis of GO and pathway of targets}

GO and KEGG analyses were performed on the targets of active components for the treatment of $A D$ using the Metascape database. The threshold $P \leq 0.05$ was set to select biological processes and pathways. The GO provides the logical structure of the biological functions, including three aspects: biological process, molecular function and cellular component, and how these functions are related to each other, manifested as a directed acyclic graph. Figure 3 showed the results of $\mathrm{GO}$ analysis for the predictive targets of the effect of Laminarin on AD, the response to steroid hormone accounted for the largest proportion in the biological process, platelet dense granule was the only one in cellular component, and steroid binding, steroid hormone receptor activity, nuclear receptor activity, transcription factor activity and direct ligand regulated sequence-specific DNA binding were at the top in molecular function.

KEGG was used to analyze the distribution of pathways for predicting the targets of Laminarin for AD. As shown in Figure 4, there were 57 enrichment pathways involved in 23 targets, including small cell lung cancer, toxoplasmosis, apoptosis, measles, hepatitis C, influenza A, tuberculosis, kaposi sarcomaassociated herpesvirus infection, Epstein-Barr virus infection, human immunodeficiency virus 1 infection and human cytomegalovirus infection. The key target proteins were enriched in the small cell lung cancer and toxoplasmosis signal pathways, further indicating the characteristics of Laminarin, multi-component and multi-pathway. The maps of small cell lung cancer and toxoplasmosis signal pathways depicted with KEGG Mapper were shown in Figure 5 and Figure 6.

\section{Discussion}


Alzheimer's disease is the 5th leading cause of death among people aged 60 years or older and no viable method has been found to prevent and cure AD. Laminaria has been reported to have a therapeutic effect on $A D$, but the mechanism is not entirely clear. Thus, the study of the Laminaria mechanism on AD is of great significance. Network pharmacology is an emerging area of pharmacology that utilizes network analysis of drug action. By considering drug actions in the context of the cellular networks, network analysis promises to greatly increase our knowledge of the mechanisms underlying the multiple actions of drugs. In this study, the possible mechanism of the treatment of AD with Laminaria was analyzed by network pharmacology.

Through database searching and screening, 7 active components of Laminaria were obtained, and 23 overlapping targets of active components and AD were collected. Through the construction and screening of the "drug-components-targets-disease" network, Saringosterol, Eckol, Eicosapnte macnioc acid, 24-

Methylenecholesterol, arachidonic acid, CLR, Fucosterol, et al. were predicted to be the active components in the treatment of $A D$ by Laminaria.

CASP3, PPARG, RELA, CCND1 and CASP9 were identified as the key genes for the treatment of AD by the PPI network. The target network indicates the characteristics of Laminarin, multi-components and multipathways. The PPI network shows that there is an interactional and complex relationship among the Laminarin target proteins. The results of GO analysis showed that the mechanism of Laminaria on AD involved biological processes like cellular processes, metabolic processes and responses to stress, cellular components such as organelles, cell membranes and cytoplasms, molecules like small molecules, cations and metal ions, and signal molecules, transcription factors, receptors, proteins, enzymes and other substances. The analysis on the target pathway showed that the main targets of Laminaria for AD were small cell lung cancer and toxoplasmosis.

\section{Conclusions}

The results suggest that Laminaria may act on multiple targets and thus play an anti-AD role. At present, there are few reports about these targets. This study may provide a new perspective for further study on the molecular mechanism of the potential targets of Laminarin in the treatment of AD. Relevant target verification experiments are in progress.

\section{Declarations}

\section{Ethics approval and consent to participate}

Not applicable

\section{Consent for publication}

Not applicable 


\section{Availability of data and materials}

All data are available in the manuscript and they are showed in figures, tables.

\section{Competing interests}

The authors declare that they have no competing interests.

\section{Funding}

This research was funded by Chongqing University of Science and Technology, grant number ckrc2019031 and the National Natural Science Foundation of Chongqing, NO. cstc2019jcyj-msxmX0083.

\section{Author Contributions}

Data curation, writing-original draft, L. Yu; Methodology, S. C. Pei; Software, K. Y. Yuan; Formal analysis, J. Zhang; Validation, J. Y. Zhao; Supervision, review and editing, project administration S. Q. Chai. All authors have read and agreed to the published version of the manuscript.

\section{Acknowledgements}

Not applicable

\section{References}

1. Isaev NK, Stelmashook EV, Genrikhs EE, Oborina MV, Kapkaeva MR, Skulachev V. P. Alzheimer's disease: An exacerbation of senile phenoptosis. Biochemistry. 2015;80:1578-81.

2. Katzman R. Alzheimer's disease as an age-dependent disorder. Ciba Found. Symp. 1988, 134, 69-85.

3. Wang J, Gu BJ, Masters CL, Wang YJ. A systemic view of Alzheimer disease - insights from amyloid- $\beta$ metabolism beyond the brain. Nat Rev Neurol. 2017;13:612-23.

4. Despres C, Byrne C, Qi H, Cantrelle FX, Huvent I, Chambraud B, Baulieu EE, et al. Identification of the Tau phosphorylation pattern that drives its aggregation. P Natl Acad Sci. 2017;114:9080-5.

5. Sudduth TL, Schmitt FA, Nelson PT, Wilcock DM. Neuroinflammatory phenotype in early Alzheimer's disease. Neurobiol Aging. 2013;34:1051-9.

6. Mancuso J, Chen YX, Zhao Z, Li XP, Xue Z, Wong STC, Optogenetic stimulation of cholinergic projection neurons as an alternative for deep brain stimulation for Alzheimer's treatment. SPIE: 2013; Vol. 8565. 
7. Chang YT, Chang WN, Tsai NW, Huang CC, Kung CT, Su YJ, Lin WC, et al. The Roles of Biomarkers of Oxidative Stress and Antioxidant in Alzheimer's Disease: A Systematic Review. BioMed Res. Int. 2014, 2014, 182303-182317.

8. Briggs R, Kennelly SP, O'Neill D. Drug treatments in Alzheimer's disease. Clin Med (Lond). 2016;16:247-53.

9. Cummings JL, Tong G, Ballard C. Treatment Combinations for Alzheimer's Disease: Current and Future Pharmacotherapy Options. J Alzheimers Dis. 2019;67:779-94.

10. Massoud F, Gauthier S. Update on the pharmacological treatment of Alzheimer's disease. Curr Neuropharmacol. 2010;8:69-80.

11. Wang J, Zhang QB, Zhang ZS, Song HF, Li PC. Potential antioxidant and anticoagulant capacity of low molecular weight fucoidan fractions extracted from Laminaria japonica. Int J Biol Macromol. 2010;46:6-12.

12. Mæhre HK, Malde MK, Eilertsen KE, Elvevoll EO. Characterization of protein, lipid and mineral contents in common Norwegian seaweeds and evaluation of their potential as food and feed. J Sci Food Agr. 2014;94:3281-90.

13. Liu M, Liu YX, Cao MJ, Liu GM, Chen QC, Sun LC, Chen HX. Antibacterial activity and mechanisms of depolymerized fucoidans isolated from Laminaria japonica. Carbohyd Polym. 2017;172:294-305.

14. Yue YY, Li ZH, Li P, Song NN, Li BQ, Lin W, Liu ST. Antiviral activity of a polysaccharide from Laminaria japonica against enterovirus 71. Biomed Pharmacother. 2017;96:256-62.

15. Lin HTV, Lu WJ, Tsai GJ, Chou CT, Hsiao HI, Hwang PA. Enhanced anti-inflammatory activity of brown seaweed Laminaria japonica by fermentation using Bacillus subtilis. Process Biochem. 2016;51:1945-53.

16. Zhu QW, Chen JH, Li Q, Wang T, Li HB. Antitumor activity of polysaccharide from Laminaria japonica on mice bearing H22 liver cancer. Int J Biol Macromol. 2016;92:156-8.

17. Oh JH, Kim J, Lee Y. Anti-inflammatory and anti-diabetic effects of brown seaweeds in high-fat dietinduced obese mice. Nutr Res Pract. 2016;10:42-8.

18. Wang J, Wang F, Zhang QB, Zhang ZS, Shi XL, Li PC. Synthesized different derivatives of low molecular fucoidan extracted from Laminaria japonica and their potential antioxidant activity in vitro. Int J Biol Macromol. 2009;44:379-84.

19. Ko SJ, Bu Y, Bae J, Bang YM, Kim J, Lee H, Beom Joon L, Hyun YH, Park JW Protective Effect of Laminaria japonica with Probiotics on Murine Colitis. Mediat. Inflamm. 2014, 2014, 417814-417824.

20. Li S, Zhang B. Traditional Chinese medicine network pharmacology: theory, methodology and application. Chin J Nat Med. 2013;11:110-20.

21. Hopkins AL. Network pharmacology: the next paradigm in drug discovery. Nat Chem Biol. 2008;4:682-90.

22. Ru JL, Li P, Wang JN, Zhou W, Li BH, Huang C, Li PD, et al. TCMSP: a database of systems pharmacology for drug discovery from herbal medicines. J Cheminform. 2014;6:13. 
23. Xu X, Zhang WX, Huang C, Yu H, Wang YH, Duan JY, Ling Y. A Novel Chemometric Method for the Prediction of Human Oral Bioavailability. Int J Mol Sci. 2012;13:6964-82.

24. Tao WY, Xu X, Wang X, Li BH, Wang YH, Li Y, Yang L. Network pharmacology-based prediction of the active ingredients and potential targets of Chinese herbal Radix Curcumae formula for application to cardiovascular disease. J Ethnopharmacol. 2013;145:1-10.

25. Stelzer G, Rosen N, Plaschkes I, Zimmerman S, Twik M, Fishilevich S, Stein TI, et al. The GeneCards Suite: From Gene Data Mining to Disease Genome Sequence Analyses. Curr Protoc Bioinformatics 2016, 54, 1.30.1-1.30.33.

26. Lu JR, Yi Y, Pan RH, Zhang CF, Han HY, Chen J, Liu WR. Berberine protects HK-2 cells from hypoxia/reoxygenation induced apoptosis via inhibiting SPHK1 expression. J Nat Medicines. 2018;72:390-8.

27. Szklarczyk D, Morris JH, Cook H, Kuhn M, Wyder S, Simonovic M, Santos A, et al. The STRING database in 2017: quality-controlled protein-protein association networks, made broadly accessible. Nucleic Acids Res. 2016;45:D362-8.

28. The Gene Ontology Consortium Expansion of the Gene. Ontology knowledgebase and resources. Nucleic acids research. 2016;45:D331-8.

29. Ogata H, Goto S, Sato K, Fujibuchi W, Bono H, Kanehisa M. KEGG: Kyoto Encyclopedia of Genes and Genomes. Nucleic acids research. 1999;27:29-34.

\section{Figures}

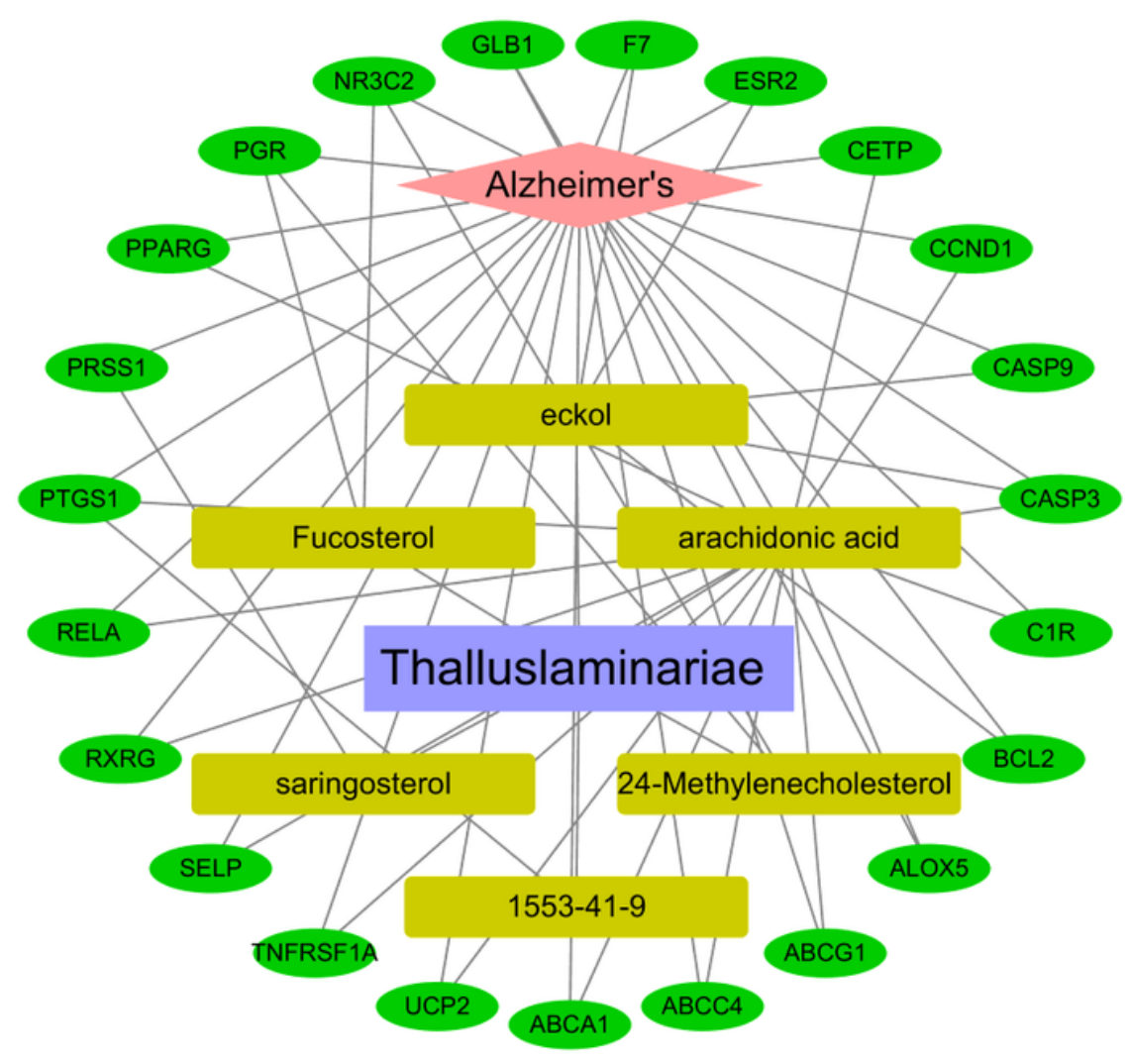


Figure 1

The "drug-components-targets-disease" visualization network.

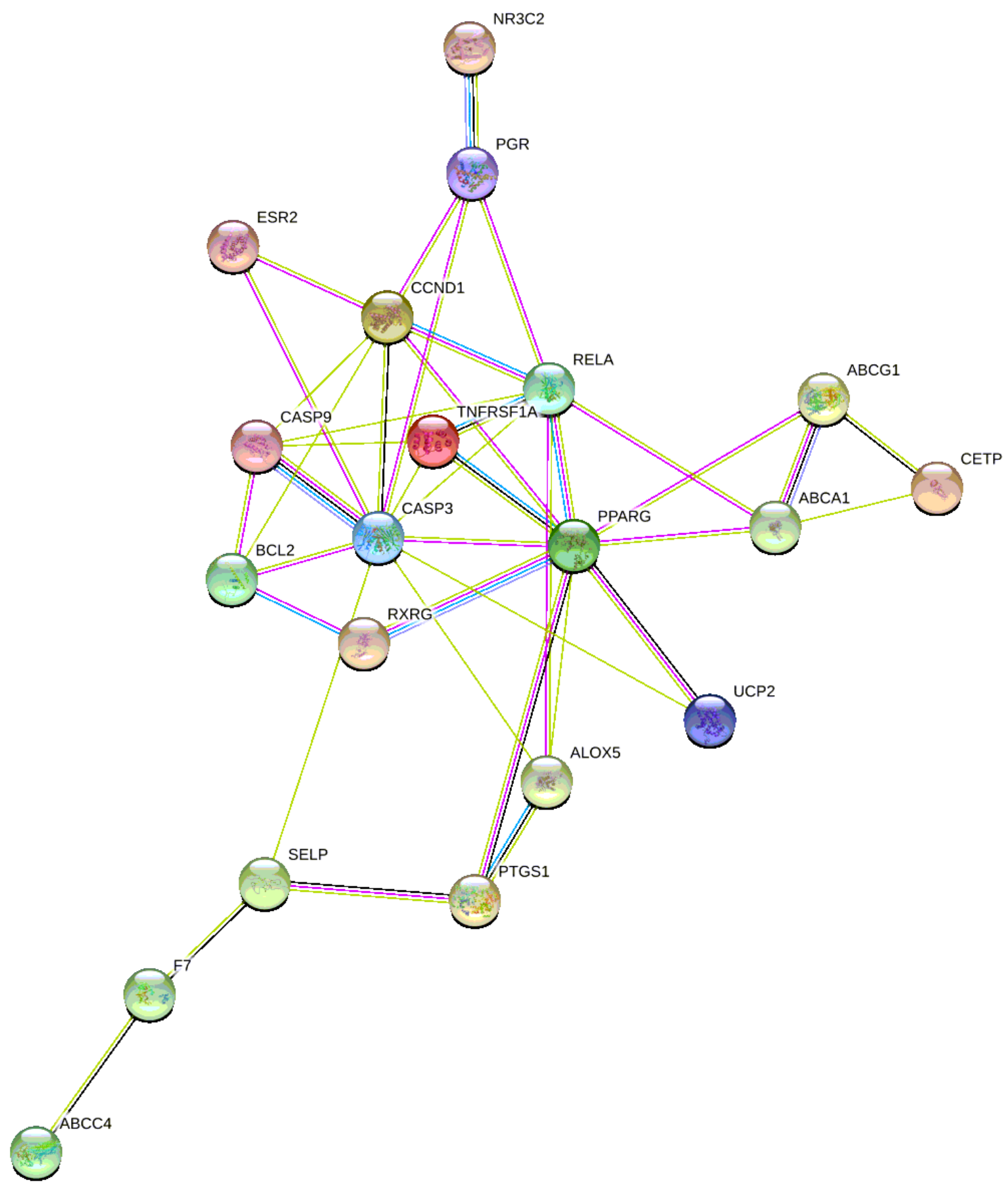

Figure 2

The PPI network of Laminarin. 


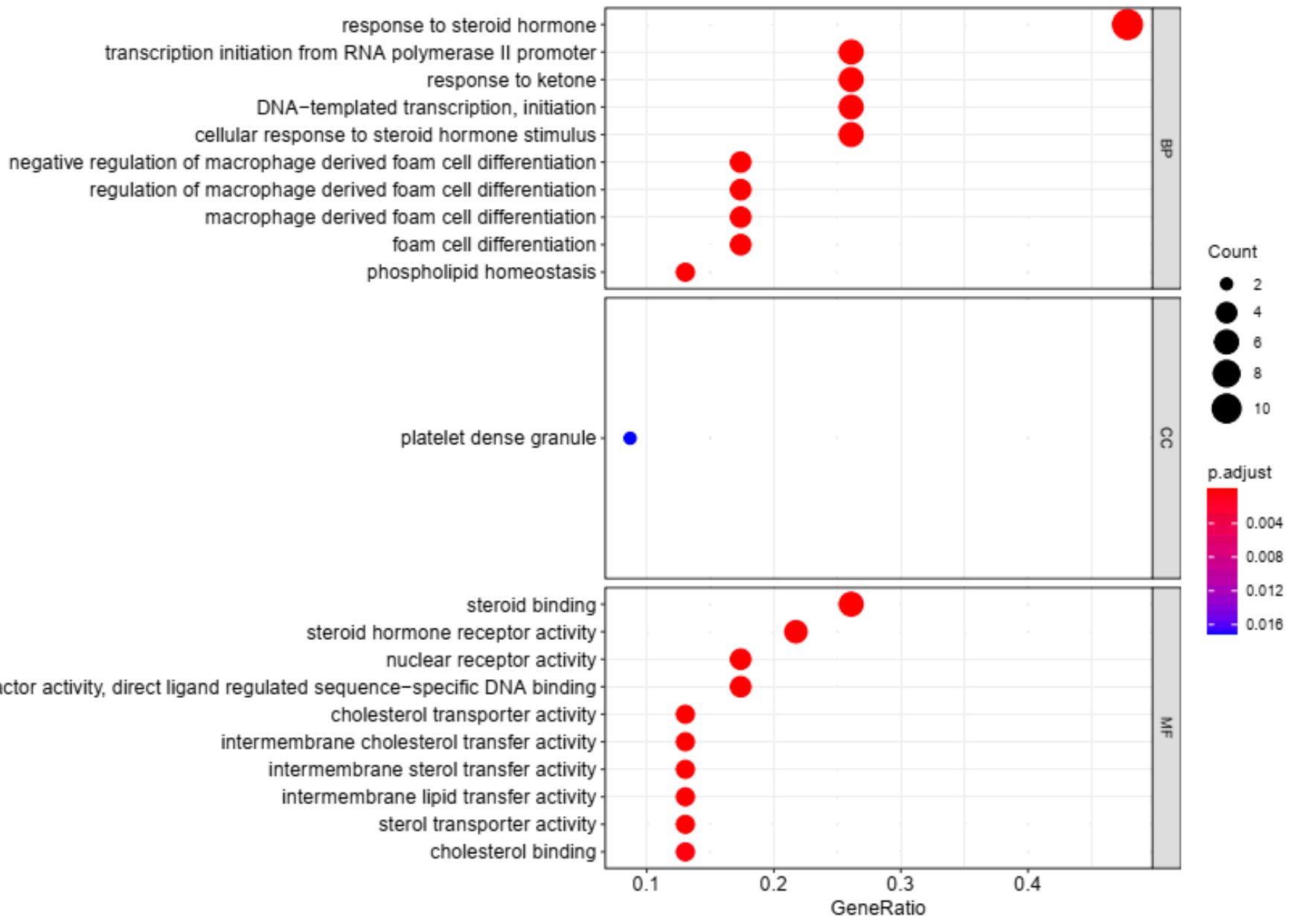

Figure 3

Enriched gene ontology terms for biological processes, cellular components and molecular functions of potential targets from the main active components of Laminarin. 


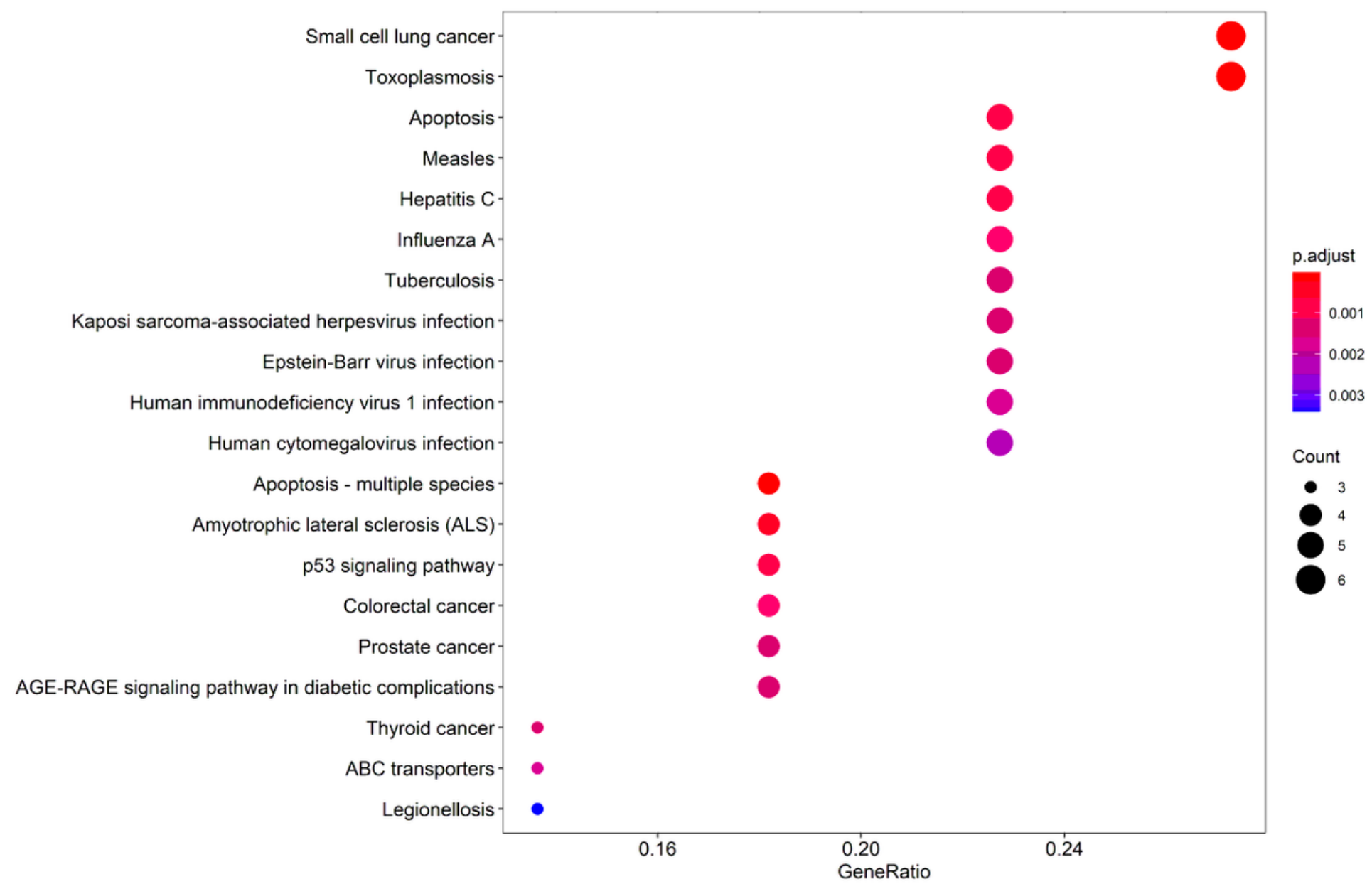

\section{Figure 4}

Enriched KEGG pathways of potential targets from the main active components of Laminarin. 


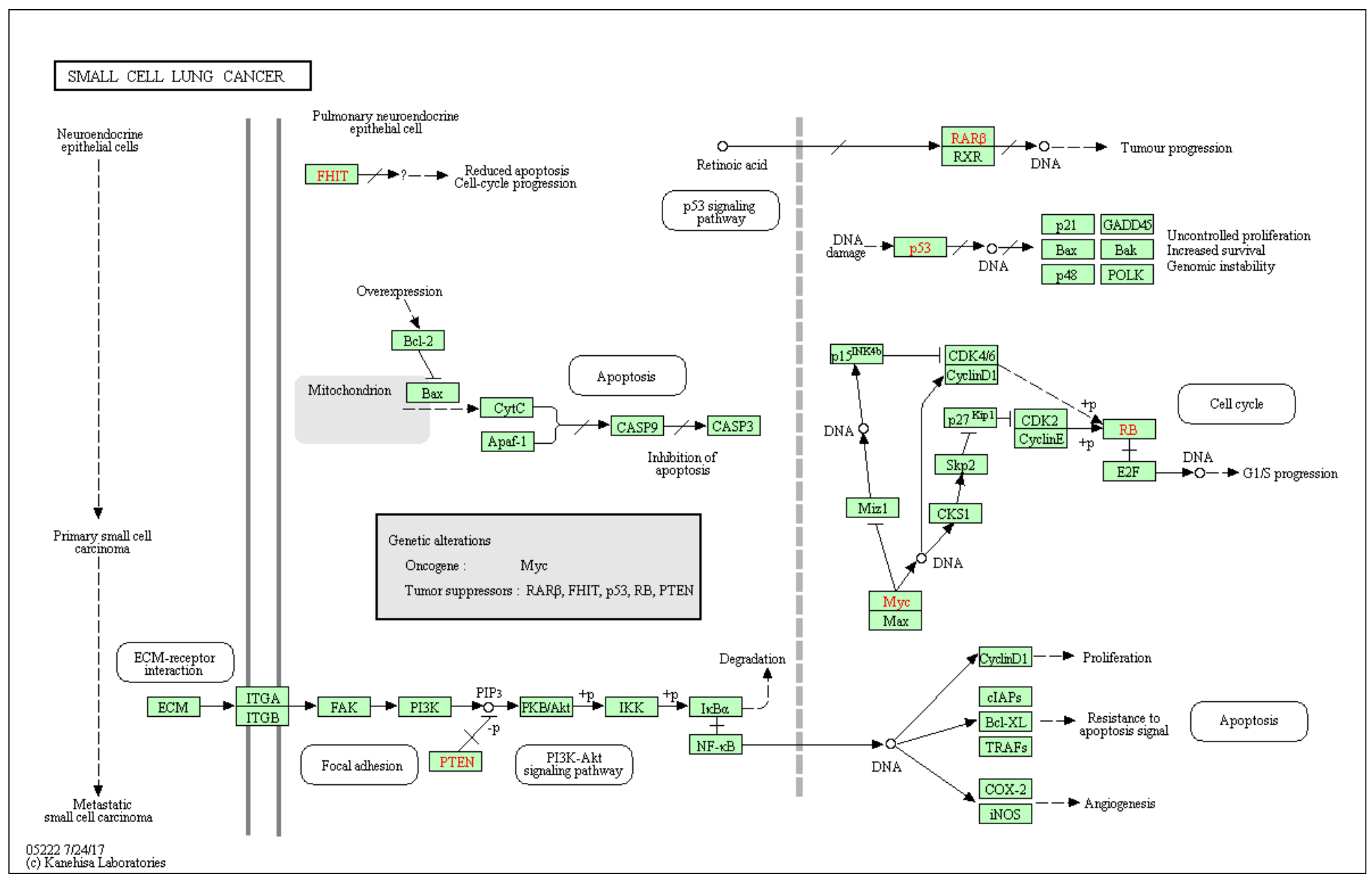

Figure 5

Signal pathway of small cell lung cancer. 


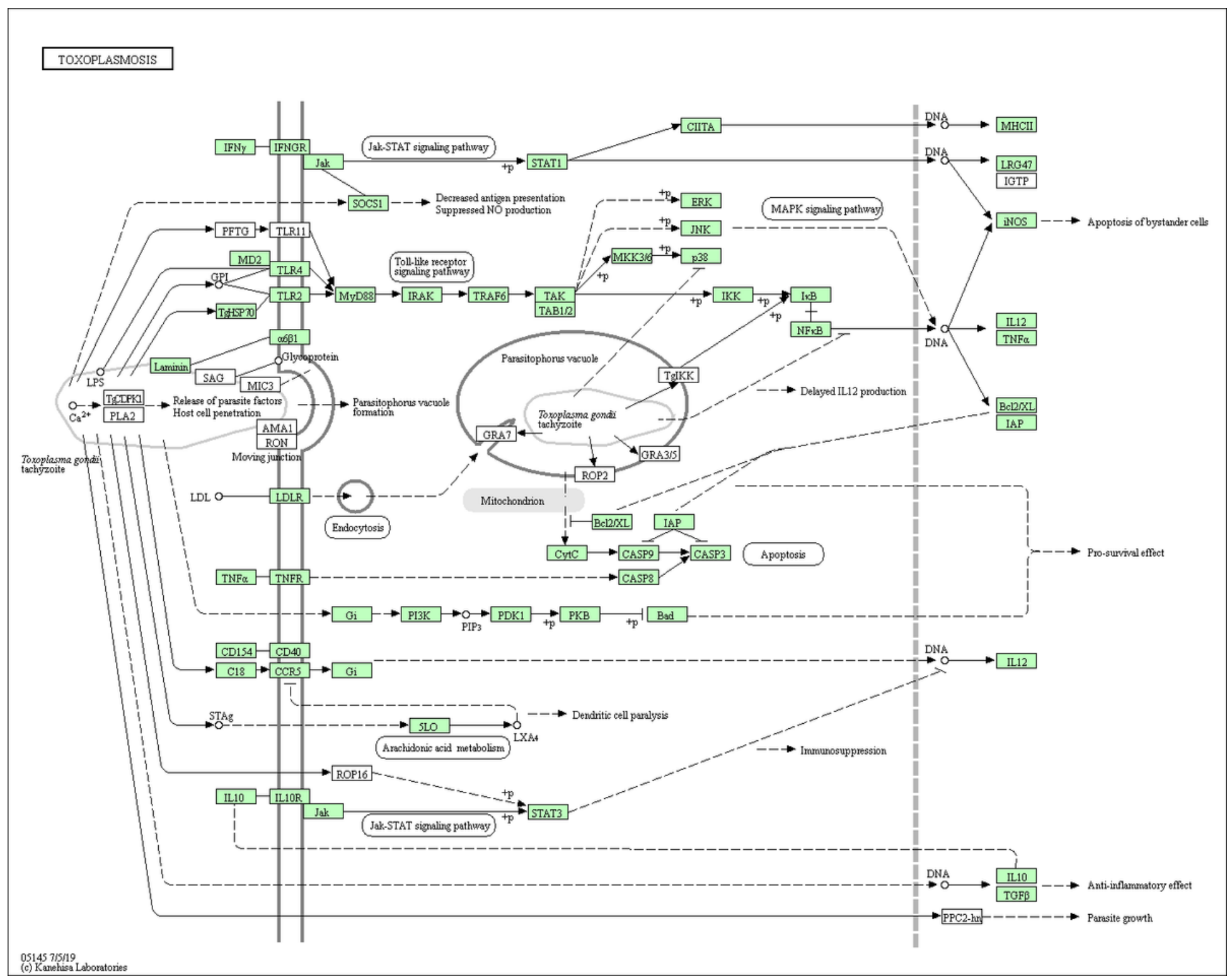

Figure 6

Signal pathway of toxoplasmosis. 